\title{
Comunidades de práctica como plataformas de mejoramiento educativo
}

\author{
Communities of Practice as Platforms for Educational Improvement
Comunidades de prática como plataformas para melhoria educacional

Milton César Flórez Cardoso* id Olga Lucia Fernández Arbeláez**

\begin{abstract}
* Candidato a Doctor en Educación. Magíster en Enseñanza de las Ciencias Exactas y Naturales. Licenciado en Matemáticas y Física. Docente de la Institución Educativa Promoción Social en Palermo (Huila). http://orcid.org/0000-0003-4721-7277 **Doctora en educación. Pasantía Posdoctoral Metodología comparada en la investigación. Docente Universitaria Universidad Católica de Manizales. Investigadora Grupo de Investigación ALFA Línea Educación y Democracia Universidad Católica de Manizales. Manizales, Caldas, Colombia. http://orcid.org/0000-0001-8110-6381
\end{abstract}

\section{OPEN ACCESS $\boldsymbol{\rho}$ \\ DOI: http://dx.doi.org/10.18634/sophiaj. 17v.1i.1104}

Información del artículo

Recibido: octubre 26 de 2020

Revisado: enero 28 de 2021

Aceptado: febrero 15 de 2021

Publicado: Abril 15 de 2021

Palabras clave: Comunidades de práctica, enseñanza, docentes, matemáticas.

Keywords: Communities of practice, teaching, teachers, mathematics.

Palavras-chave: Comunidades de prática, ensino, professores, matemática

Cómo citar: /how cite:

Flórez, M.C., Fernández, O.L (2021) Comunidades de práctica como plataformas de mejoramiento educativo. Sophia 17 (1), e1104

Sophia-Educación, volumen 17 número 1. enero/junio 2021. Versión español

\section{RESUMEN}

El presente artículo da cuenta de una revisión bibliográfica sobre comunidades de práctica cultivadas en el contexto educativo. Su propósito es servir de base documental, para la visualización de un panorama preliminar, en el posterior desarrollo de una investigación sobre los efectos que tiene la configuración de una comunidad de práctica en la enseñanza de las matemáticas, cuando es conformada por docentes de educación básica primaria de una misma institución educativa. Por lo que se presentan los principales hallazgos de investigaciones relacionadas con tres niveles de la educación colombiana, a saber: la universidad y la educación básica secundaria y media, donde se evidencian una mayor cantidad de investigaciones al compararse con la educación básica primaria. Una de las principales conclusiones, es que las comunidades de práctica desempeñan un papel fundamental, cuando se utilizan para mejorar los procesos educativos permitiendo fortalecer los mecanismos de mejoramiento de las prácticas pedagógicas. 
Copyright 2021 Universidad La Gran Colombia

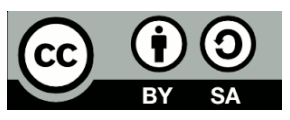

Conflicto de interés:

Los autores declaran no tener ningún conflicto de interés.

Correspondencia de autor:

milton.florez@ucm.edu.co ofernandez@ucm.edu.co

\section{ABSTRACT}

This article reports a literature review on communities of practice developed in the educational context. Its purpose is to serve as a documentary basis for the visualization of a preliminary panorama in the subsequent development of a research on the effects of the configuration of a community of practice in the teaching of mathematics, when it is formed by elementary school teachers from the same educational institution. Therefore, the main findings of research related to three levels of Colombian education are presented, namely: university and basic secondary and middle school education, where a greater amount of research is evidenced when compared to basic elementary education. One of the main conclusions is that communities of practice play a fundamental role when they are used to improve educational processes, allowing the strengthening of mechanisms for the improvement of pedagogical practices.

\section{RESUMO}

Este artigo relata uma revisão bibliográfica sobre comunidades de prática cultivadas no contexto educacional. Tem por finalidade servir de base documental, para a visualização de um panorama preliminar, no posterior desenvolvimento de uma investigação sobre os efeitos que a configuração de uma comunidade de prática tem no ensino da matemática, quando este é constituído por professores de educação, escola primária da mesma instituição de ensino. Portanto, são apresentados os principais resultados das pesquisas relacionadas aos três níveis da educação colombiana, a saber: a universidade e a educação básica secundária e média, onde se evidencia uma maior quantidade de pesquisas quando comparada à educação primária básica. Uma das principais conclusões é que as comunidades de prática desempenham um papel fundamental, quando são utilizadas para a melhoria dos processos educativos, permitindo fortalecer os mecanismos de melhoria das práticas pedagógicas. 


\section{Introducción}

Las comunidades de práctica (CoP) son un tipo de estructura organizacional que parecen haber surgido en el sector empresarial. Sin embargo, uno de sus principales voceros en la actualidad menciona que: "Aunque el término puede ser nuevo su experiencia no lo es" (Wenger, 2001, p. 24). Pues todos los seres humanos pertenecemos de una u otra manera a diversas comunidades de práctica. Ejemplos de ellas son las formadas por los miembros de un hogar o las que brotan entre compañeros de trabajo en una fábrica. Pues en su interior se establecen rituales, formas de ser y de actuar que corresponden a unas pautas determinadas; también, existe una cantidad de miembros específica que puede variar, por ejemplo, por el nacimiento de un nuevo integrante en la familia, o por el despido o contratación de un operario. Poseen desafíos compartidos como ¿Qué hacer para que el dinero alcance hasta fin de mes? o ¿Cómo cumplir con una meta de productividad? En ambos casos cada miembro de la colectividad se involucra en la medida de su responsabilidad o bajo un acuerdo (en algunos casos, tácito y en otros, previamente establecido mediante un contrato).

Un ejemplo de CoP suele resultar cuando su filiación no es familiar ni laboral, sino educativa. Cuando sus miembros pertenecen a una escuela o están ligados a algún contexto formativo, tanto si son docentes, como si son estudiantes, o si hacen parte del personal de directivos docentes. Todos están inmersos en un conjunto de normas o reglamentos diseñados (generalmente) sobre principios de calidad que caracterizan lo que sucede en su interior y que muy seguramente tienen como objetivo principal desarrollar aprendizajes efectivos en sus estudiantes. En su libro Comunidades de práctica: aprendizaje, significado e identidad, Wenger (2001) propone replantear el aprendizaje desde la perspectiva de la participación, ya sea como individuo, como comunidad o como organización, pues supone que en todo momento se aprende como parte integral de la vida cotidiana.

Aunque las CoP se encuentran en innumerables contextos y sirven favorablemente a todo tipo de organizaciones, para el propósito del presente artículo se muestra una revisión bibliográfica de las investigaciones relacionadas con comunidades de práctica en el ámbito de la enseñanza de las matemáticas. Desde allí, fue posible visibilizar las características de su puesta en marcha, en tres niveles: universidad, educación secundaria y media y educación primaria. Y ante lo cual se establece como primera medida, un marco teórico que sustenta la revisión documental.

\section{Caracterizando las comunidades de práctica}

Las Comunidades de Práctica (CoP) según (Wenger, McDermott \& Snyder, 2002) están conformadas por: "Personas que comparten una preocupación, un conjunto de problemas, o una pasión por un tema, las cuales, profundizan su conocimiento y experiencia en esta área interconectándose de manera continua" (p. 5). Hoy a través de su estudio y revisión se pueden diferenciar tres elementos que las constituyen (ver figura 1), a saber: un compromiso mutuo, una empresa conjunta y repertorio compartido.

Figura 1. Elementos que costituyen las comunidades de práctica
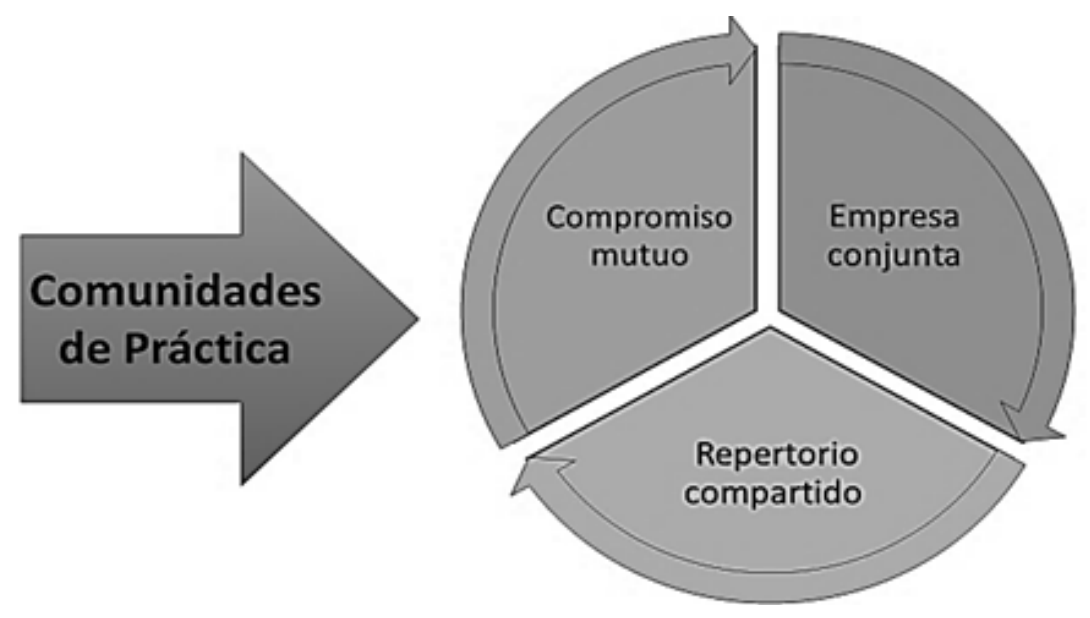

Fuente: elaboración propia a partir de Wenger (2001)

Estos tres aspectos particularizan a las comunidades de práctica y es posible establecer una correlación entre ellos y un grupo de profesionales como los docentes de un establecimiento educativo. Se pretende utilizar este tipo de estructura social como una forma de vehicular las principales preocupaciones de los profesores de 
primaria hacia una ruta de progreso, que se intentará desarrollar ya no en solitario, sino en equipo. Ese grupo unirá sus ideas, sentires, expectativas y tratará de brindarles atención en conjunto. Además se compartirán modos de hacer, que al difundirse entre pares potencializarán el abanico de apuestas pedagógicas que se encontrarán a disposición de los docentes que estén prestos a buscar diferentes formas de innovar sus prácticas de aula para mejorar los aprendizajes de los estudiantes.

Las oportunidades que surgen de este constructo social son valiosas para el sector educativo, pues proporciona una plataforma que colabora en la gestación y puesta en marcha de propuestas de mejoramiento encaminadas a empoderar a los docentes mediante el intercambio de saberes adquiridos durante su práctica docente y enriquecidos por el trabajo en equipo. Cuando se analiza desde estos tres pilares es posible entrever las oportunidades que surgen al combinarlos, de manera que se articulen en una meta común. Así la empresa conjunta se relaciona con la misión del educador que desea guiar un proceso formativo en sus estudiantes, mientras que el compromiso mutuo tiene que ver más con la característica de la afiliación al grupo que invita a los integrantes a participar de un convenio de trabajo mancomunado, y, el repertorio compartido se manifiesta en la reflexión y construcción de metodologías atractivas para la enseñanza de las matemáticas escolares.

Zea y Acuña (2017) definen las CoP como un sistema de organización flexible que favorece el intercambio de conocimientos y experiencias en un ambiente donde las interacciones y la cooperación enriquecen los contextos de aprendizaje. De acuerdo con Giraldo \& Atehortúa las comunidades de práctica se presentan:

Como una estrategia de gestión del conocimiento que permite la integración, la comprensión, el compromiso mutuo y repertorio compartido de recursos intangibles, que es el resultado de una práctica compartida de las personas y de sus conocimientos desde la conformación espontánea de dicha comunidad (Giraldo \& Atehortúa, 2010, p. 143)

Es McDermott (1999) quien menciona que: "La llave para conducir el cambio hacia la compartición del conocimiento se encuentra probablemente en las comunidades de práctica” (p. 116). Esta afirmación generó gran interés por estudiar de qué forma podría estructurarse una propuesta encaminada al mejoramiento de la enseñanza de las matemáticas usando esta configuración organizacional. En este sentido, Pérez (2011) menciona que las comunidades de práctica ofrecen una oportunidad: "Para aprender de otra manera, participando y apropiándose del conocimiento distribuido" (p. 26). Así al interior de este tipo de organismo se favorece la interacción entre individuos que buscan resolver problemas, facilitando el acceso a cada integrante de la comunidad sobre el acervo de conocimientos que cada miembro posee y que pone al servicio de sus compañeros. Es decir, el nivel de aplicación de las comunidades de práctica tiene que ver: "con que un grupo de personas, con intereses comunes, examine un problema concreto y decida actuar para solucionarlo involucrando saberes del orden ético, moral y político" (Barragán, 2015, p. 173).

De acuerdo con Godói de Sousa y Eiko (2013) las CoP constituyen una de las estructuras más valiosas de las organizaciones que consideran el conocimiento como un recurso central, donde el pensamiento libre es un capital fundamental. Al respecto, Jubert (1999) afirma que esta nueva forma de organización que está emergiendo "promete complementar las estructuras existentes y rápidamente impulsar el conocimiento, el intercambio y el aprendizaje" (p. 24). Estas investigaciones apuntan hacia un horizonte de constitución de posibilidades de apoyo al sistema educativo, pues de acuerdo con Vásquez (2011), las comunidades de práctica: "Han demostrado ser estructuras en las cuales el conocimiento se comparte y circula" (p. 21), y de manera similar, Rodríguez (2007) afirma que las comunidades de práctica serían una variante clave a considerar "para comprender los procesos y mecanismos de influencia educativa que se dan dentro de las instituciones formales, y a los que tradicionalmente la reflexión pedagógica solo había sabido nombrar, pero no comprender en su modo de actuar" (p. 12).

Lo anterior aunado al fenómeno del "pensar juntos" en comunidad proporciona una comprensión más profunda de los elementos estructurales de las comunidades de práctica de Wenger (2001) y ello desarrolla: "La creación de vías para reflexionar en forma regular sobre los problemas de la vida real que a las personas realmente les interesan" (Pirko et al, 2016, p. 15). Este elemento reflexivo abarca innumerables significados sobre los que se ha teorizado en profundidad, así Perrenoud (2011) afirma que la práctica reflexiva cuando ocurre durante la acción "consiste en preguntarse lo que va a pasar, lo que podemos hacer, lo que hay que hacer y cuál es la mejor táctica; qué orientaciones y qué precauciones hay que tomar, qué riesgos existen” (p. 30), mientras que si la práctica reflexiva ocurre sobre la acción remite a "tomar la propia acción como objeto de reflexión... y si la acción se cumple, permite entonces, comprender, aprender, integrar lo que ha sucedido” (p. 31). Podría 
decirse que una práctica es reflexiva si se convierte en una forma de existencia al interior de una organización, movilizando y adoptando medidas que aportan un sentimiento de preparación y coherencia sobre lo que pueda emerger.

Por su parte Cubero y Sazatornil (2013), encuentran que fruto de reflexionar en una comunidad de práctica se deriva en un "tipo de relación horizontal en la que existe respeto y escucha mutua, que permite que los participantes se sitúen como interlocutores válidos" (p. 424) donde el experto y demás integrantes "asumen la tarea de establecer un conocimiento compartido al fijar metas comunes" (p. 425). Por ello, los cambios que realmente logran algún tipo de transformación de las prácticas de aula son los que se producen a partir de la reflexión del docente entre el acto de enseñar y el contexto en el que se desenvuelve el proceso educativo.

Las CoP son utilizadas no solo para formar o actualizar educadores en ejercicio, también se usan para reproducir procesos de autoconocimiento (Agudelo y Lovera, 2017), por lo que podrían extender un puente comunicativo entre la reflexión individual y la reflexión en comunidad dirigido a mejorar la enseñanza de las matemáticas, pues según Castellanos (2015), solo es posible mejorar la práctica docente si los profesores cambian sus actitudes y comportamientos al desempeñar su ejercicio. Así que, lo que brinda este tipo de organización social, es una plataforma de transformación que gestiona el fortalecimiento pedagógico en la escuela. Aunque, siempre es necesario tener presente que no solo sea utilizada para intercambiar conocimientos prácticos derivando en un uso puramente instrumental (Trust, 2015) desperdiciando la construcción de identidades que generen lazos significativos entre sus miembros como señala Wenger (2001).

\section{Metodología}

El presente artículo de revisión muestra la búsqueda sistemática que da cuenta de un análisis documental vinculado a dos elementos fundamentales e interconectados en la presente investigación. Para conseguirlo, se han analizado los artículos de investigación, libros, ponencias resultantes de investigaciones, tesis de maestría y doctorado sobre Comunidades de Práctica existentes en el ámbito educativo y en especial, las relacionadas con el área de matemáticas. Por lo que se utilizaron las bases de datos de Scopus, Google Scholar, Scielo, Dialnet y ERIC, con el ánimo de determinar las profundidades alcanzadas y los desafíos generados. Se procedió a delimitar la revisión bibliográfica a tres de los niveles de la educación pública en Colombia, a saber: la educación universitaria, la educación secundaria y media y la educación primaria. En la siguiente tabla se muestra detalladamente los documentos hallados y analizados al respecto.

Tabla 1. Documentación del proceso de búsqueda

\begin{tabular}{|c|c|c|c|c|c|}
\hline \multirow[b]{2}{*}{ Relación } & \multicolumn{5}{|c|}{ Tipo de Documento } \\
\hline & $\begin{array}{c}\text { Artículo } \\
\text { Científico }\end{array}$ & Ponencias & $\begin{array}{c}\text { Tesis } \\
\text { Maestría }\end{array}$ & $\begin{array}{c}\text { Tesis } \\
\text { Doctorado }\end{array}$ & Libro \\
\hline $\begin{array}{l}\text { Marco } \\
\text { Teórico }\end{array}$ & 11 & 4 & - & - & 3 \\
\hline $\begin{array}{l}\text { Educación } \\
\text { Superior }\end{array}$ & 15 & 6 & - & - & 2 \\
\hline $\begin{array}{l}\text { Educación } \\
\text { Secundaria }\end{array}$ & 19 & 3 & - & 3 & - \\
\hline $\begin{array}{l}\text { Educación } \\
\text { Primaria }\end{array}$ & 2 & 4 & 1 & - & - \\
\hline Total & 47 & 17 & 1 & 3 & 2 \\
\hline Gran total & & & 70 & & \\
\hline
\end{tabular}

Fuente: elaboración propia 


\section{Comunidades de practica en contextos educativos}

\section{En la educación universitaria}

La construcción de CoP en la educación universitaria (también conocida como educación superior en Colombia) refiere en primera instancia, por caracterizar a los docentes enfocándose en lo que piensan que es relevante y significativo en el desarrollo de sus prácticas (Morales y Flores, 2013). Aunque, es necesario tener en cuenta las aportaciones de los participantes en igual medida, así como estimular la confianza, que permitan germinar un sentimiento de apropiación mutua de una iniciativa proyectada (Morueta et al, 2009).

Algunos autores como Bozu e Imbernon (2009) afirman que:

crear CoP que potencien la colaboración y la cooperación entre el profesorado y el intercambio de conocimiento práctico profesional, se perfila como una de las mejores alternativas para un modelo de formación del profesorado orientado hacia la creación de espacios de reflexión, formación e innovación pedagógica" (p. 5).

De acuerdo con estos autores las CoP apalancan los procesos de mejoramiento de la enseñanza al interior de los establecimientos educativos. Así desde una perspectiva operativa las CoP se encargarían entre otros aspectos de:

- Detectar experiencias exitosas a fin de que se sistematicen y socialicen como parte del desarrollo del currículo.

- Generar espacios de reflexión sobre la práctica docente.

- Nutrirse con las experiencias de otros maestros (Almánzar, 2013, p. 80).

Es interesante observar que las CoP brindan una armazón que tiene potencialidades de sostener el mejoramiento de los procesos de enseñanza aprendizaje, tal como lo sostiene Camargo (2010) quién complementa esta idea al afirmar que "es posible utilizar las comunidades de práctica en la enseñanza para modificar la manera tradicional donde hay un maestro que sobresale y unos estudiantes que se supone aprenden". (p. 373). Esto destaca dos elementos importantes, a saber: las características que poseen las comunidades de práctica en la educación universitaria y la particular aplicación que de ellas podría hacerse en la enseñanza de las matemáticas. Esta idea es robustecida por Ávalos (2011) cuando expresa que una "comunidad de práctica puede producir un proceso casi simbiótico entre la generación de conocimiento a través de la experiencia de colaborar en torno a temas o a situaciones concretas y el surgimiento de liderazgos que hacen posible su efectividad" (p. 250). Estos elementos muestran que la puesta en marcha de las CoP aportan elementos metodológicos para el mejoramiento de la educación universitaria. Su desafío es el de "pensar en espacios reales y significativos que ayuden a representar la enseñanza y su complejidad para la toma de buenas decisiones" (Ravanal, 2016, p. 23).

Algunas de las investigaciones sobre CoP desarrolladas en ámbitos universitarios están dirigidas a fortalecer el proceso de formación y seguimiento a maestros en ejercicio de la educación básica secundaria. Para Bozu e Imbernon (2009) dichas CoP se han convertido en "un espacio de intercambio de visiones, experiencias y prácticas que han facilitado un acercamiento entre profesores nóveles y experimentados y una reflexión compartida y personal sobre la enseñanza" (p. 5). Este intercambio permite el flujo de información en doble dirección, de manera que los sujetos son transformados en el acto de interactuar constantemente. Para Cordero (2013) estas interacciones entre docentes expertos y noveles promueven "la observación, el diálogo, la experimentación a través del ensayo-error, el ejercicio planificado y regulado de la reflexión en y sobre la práctica" (p. 833). Para Solomon et al (2015) es necesaria la retroalimentación de docentes mentores hacia futuros docentes pues nutre el proceso de consejería grupal que promueve el fortalecimiento pedagógico del novato.

En otros enfoques investigativos se observa una especial orientación a revisar el papel de la reflexión docente en la práctica de las comunidades. Para Shön (1982) la reflexión realizada desde la acción permite la toma de conciencia del individuo, y este, es un elemento indispensable en la transformación de las prácticas. En efecto, Córdoba (2013) afirma que la generación de espacios reflexivos en las CoP posibilita el fortalecimiento individual y colectivo que los llevará a superar cualquier obstáculo, y, en esa misma línea, Chacón (2006) comenta que el uso de CoP en la educación universitaria promueve estrategias de enseñanza reflexiva que consolidan la autonomía de la comunidad. De esta forma las CoP impulsan el mejoramiento del desempeño 
docente promoviendo una gestión de calidad alrededor de sus prácticas. Pues, para Roig y Rivera (2008) la constitución de una CoP promueve el ambiente individual y grupal adecuado para involucrarse en reflexiones encaminadas al mejoramiento de sus prácticas educativas y en consecuencia para alcanzar aprendizajes con entendimiento de sus estudiantes.

Para algunos otros investigadores es muy importante analizar el papel de las Cop en la formación relacionada con la práctica, con el ánimo de trazar rutas de cualificación pedagógica. De este modo, Ramos y Manrique (2015) indican que los miembros de la CoP expresan su interés por profundizar en los contenidos abordados y transformados en el mismo proceso educativo al adoptar la perspectiva de usar las prácticas de aula como elementos de aprendizaje entre pares. Esta visión según Da Silva (2010) fomenta que la evaluación de una clase dada sea revisada en conjunto realizándole las adaptaciones que se consideren necesarias bajo un proceso de negociación de significados que resulte en un repertorio común de los diferentes modos de intervenir en el aula. Vargas et al (2019) fortalecen esta posición al afirmar que las CoP enriquecen el diálogo interdisciplinar al estimular el trabajo colaborativo entre pares académicos, ya que se mejora el proceso de formación del docente y se convierte en una plataforma de transversalización de los saberes a enseñar.

Sabulsky (2016) indica que la CoP tiene la potencialidad de servir "como ámbito propicio para perder el miedo y ganar confianza ante una tarea que el profesor debía enfrentar posiblemente solo en el aula” (p. 8). Este aspecto señala serios indicios de que las CoP potencian la buena enseñanza y sirven para visualizar rutas de implementación para el mejoramiento escolar. Aunado a esto, Ortega y Hernández (2015) mencionan que es "viable constituir a futuro nuevas CoP pues posibilita a los docentes una vía de mejora en su quehacer diario, además de representar la oportunidad de trascender en su trabajo cotidiano dentro y fuera del aula" (p. 91); considerando que "al fomentar las comunidades de práctica en los establecimientos educativos se lograrán mejores propuestas de aula con enfoques más holísticos y contextuales que contribuyan a formar ciudadanos reflexivos y participativos" (Edwars y Rebollo, 2010, p. 13). Por lo tanto, es plausible destacar que la práctica desarrollada en comunidades "posibilita momentos de reflexión y de discusión, de compartir experiencias, de creación de vínculos afectivos y de respeto mutuo, así como rasgos de desarrollo profesional y de constitución de la identidad del profesor". (Rodrigues y Manrique, 2015, p. 985).

En cuanto a los enfoques investigativos utilizados la mayoría de autores manifiestan su inclinación por la investigación cualitativa. De allí que autores como Anguita et al (2006) afirmen que "esta le permite desarrollar herramientas para el trabajo de aula en las clases, así como para investigar las mismas" (p. 162). En un contexto similar, la investigación desarrollada por Aydee (2015) también acude a una perspectiva cualitativa con un encuadre teórico metodológico de investigación narrativa de corte hermenéutico y narrativo con una estrategia de análisis de contenido. Mientras que Cordero (2013) investiga desde una perspectiva interpretativa desarrollando una lógica de generación conceptual a través de un estudio de caso buscando "aprehender la complejidad del mismo sin ánimo de establecer generalizaciones, sino entendiendo a este tipo de indagación como motor de la reflexión sobre la temática y la comprensión del caso en estudio" (p. 832). Por su parte Roig y Rivera (2008) promueven como objetivo principal de la CoP la enseñanza de la metodología investigativa de investigación acción a medida que la utilizan en el desarrollo de las actividades.

Este recorrido investigativo evidencia el enorme acerbo de posibilidades que ofrece el uso de las CoP en la universidad. Es más, este tipo de estructura social podría "evolucionar a una comunidad de investigación y práctica en la medida que más y más profesionales e investigadores se involucren con la comunidad, la influencia que ejerza se transformará en su continuo crecimiento" (Cheek et al, 2009, p. 237). Lo que contribuiría de acuerdo con Morales y Flores (2013) a reconocer el acto educativo como "un hecho social en el que la participación da lugar a un proceso de aprender y construir conocimiento" (p. 117). Incluso, este tipo de procesos es posible desarrollarlos en las CoP en línea, pues, según Ekici (2017) a través de la participación activa de los docentes es factible desarrollar habilidades como el pensamiento crítico, que apalanca los procesos de transformación educativa. No obstante, es importante tener en cuenta que en este tipo de CoP pueden existir grandes diferencias de nivel y los participantes pueden tener dificultades en el manejo de macro grupos de trabajo (Torres y Soriano, 2015).

\section{En la educación básica secundaria y media}

En la educación básica secundaria también se han realizado investigaciones que hacen hincapié en las ventajas que tiene el uso de las CoP para apalancar procesos de mejora en diversos contextos relacionados con la enseñanza. Para Bustos y Moreno (2008) gracias a las reflexiones al interior de la CoP de profesores que 
enseñan matemáticas en la educación básica, "se accesa sobre el currículo y demás procesos educativos con poder decisorio, analizando la correspondencia entre los planteamientos curriculares y lo que efectivamente ocurre en una práctica docente, es decir, se hace posible acoplar teoría y práctica" (p. 5). Por su parte, Parada (2011) expone que uno de los hallazgos más importantes al investigar sobre CoP "lo constituye el aporte teórico y práctico que permite orientar los procesos de reflexión por parte de los profesores antes, durante y después de sus clases" (p. 262). En el decir de Murcia y Bustos (2000) "permite a los docentes de secundaria dotar de sentido un conocimiento dándole valor a través de la elaboración de significados que pueden lograrse por medio de negociación al interior de las comunidades de práctica" (p. 7), es decir, que las comunidades de prácticas de docentes permiten a sus integrantes dar un nuevo significado a las actividades de enseñanza y aprendizaje mediante la integración de variados y nuevos procesos de aplicación de las dinámicas educacionales.

En la investigación de González et al (2013) hallaron que las CoP en la educación básica secundaria:

Fomentan la comunicación continua y pertinente entre los compañeros profesores, buscan una mejor comprensión de sus prácticas y de las de los demás propiciando que el intercambio se desarrolle en un ambiente de respeto, apertura, madurez para recibir y hacer críticas constructivas (p. 110)

Este elemento alienta la generación de iniciativas renovadoras al interior de una institución educativa, pues a través de la cohesión que produce el tener objetivos comunes se visualiza un camino de construcción de soluciones desde el interior de los establecimientos respaldado por una organización que une voluntades y propone alternativas ajustables a cualquier contexto particular.

Un beneficio que plantea Valdés (2010) es que si:

Se busca fomentar en los alumnos la colaboración, la comunicación, la interacción y la socialización, primero deben promoverse estas, entre los profesores y que mediante la creación de una CoP de profesores puede motivar a los docentes para que colaboren en un proceso de cambio educativo, y a la vez, buscar que los resultados positivos que se generen de esta agrupación perduren. Si la colaboración docente es continua y duradera, los resultados en el aprendizaje de los alumnos serán cada vez mejores" (p. 6)

Lo anterior es evidencia de que las comunidades de práctica transforman la práctica educativa, puesto que las competencias profesorales serán desarrolladas de acuerdo a las individualidades de los docentes y a sus subjetivas formas de enseñanza que se integran mediante la socialización permanente en cada espacio de encuentro de las CoP y que enriquecen el aprendizaje de los docentes que las componen modificando las dinámicas de la enseñanza y del aprendizaje en las aulas. Al respecto, González (2014) refiere que en las comunidades de práctica "el docente es protagonista de su propia formación" (p. 196); cuestión que habilita el empoderamiento de los maestros para que se reconozca como un elemento generador de apuestas pedagógicas. Un movilizador de herramientas de mejoramiento educativo.

Desde la perspectiva de la formación docente Cyrino (2016) comenta que las propuestas que valoran las experiencias, los repertorios y el conocimiento de los involucrados, así como el desarrollo de aprendizajes a través de negociaciones de significado, son las más adecuadas para el proceso de cualificación docente. En tal sentido Conde et al (2017) argumentan que "el análisis de la participación en el repertorio de prácticas proporciona elementos para caracterizar cómo una CoP puede influir en el desarrollo profesional de los profesores". (p. 456). Y es que para apoyar el desarrollo sostenible de un grupo de profesores se requiere una mentalidad abierta al cambio, que posibilite el intercambio y retroalimentación de opiniones estimulado al interior de las CoP (Jho et al, 2016). Así, el proceso de aprendizaje se realiza en conjunto "generando interacciones entre los miembros de la propia comunidad mediante debates, prueba de que una aproximación social en la formación docente se puede realizar a través de CoP" (Sanz y Pantoja, 2015, p. 125). Sin embargo, la participación en dichas comunidades "será ineficaz si no va acompañada de una determinación de cambiar por parte del docente y de implantar nuevas maneras de realizar su práctica en el aula” (Vallín, 2013, p, 71).

Por esta razón es importante la existencia de líderes dentro del grupo que al ser aceptados por la CoP asuman "la función de unificar las acciones, distribuir la información, organizar el trabajo y velar por un correcto funcionamiento de la evolución del grupo (García et al, 2008). Estas funciones se hacen explícitas cuando "cada uno de los miembros actúa como maestro y tutor de los otros, planifican actividades de la comunidad y desarrollan herramientas y marcos de referencia que forman parte del conocimiento común de la comunidad (Vargas el al, 2016). Por lo que el liderazgo dentro de las CoP "vuelve explícita la transferencia informal de 
conocimiento dentro de redes y grupos sociales ofreciendo una estructura formal que permite adquirir más conocimiento a través de las experiencias compartidas dentro del grupo" (Mayorga, 2014).

Otro elemento importante a tener en cuenta en este tipo de estructura según Graven (2004) es el desarrollo de la confianza, pues según él, la confianza debería ser un aspecto de la teoría social del aprendizaje de Wenger (explícito en las CoP) pues, "junto con el significado, la práctica, la identidad y la comunidad, permitiría estimular el liderazgo que posibilite el desarrollo de aprendizajes en el interior de la CoP" (p. 2). El factor de la confianza pone sobre la mesa las relaciones humanas como primordial elemento gravitatorio en la construcción y puesta en marcha de las organizaciones sociales, en especial, aquellas en las que se comparte no solo maneras o formas de hacer las cosas, sino también donde se exponen visiones personales sobre los modos de ser docente o enseñante de matemáticas, un área para la que, en la mayoría de los casos, ningún profesor de primaria en Colombia ha sido instruido formalmente, por mencionar solo un ejemplo. Es relevante indicar que, en un ambiente escolar caracterizado por la confianza:

El surgimiento del talento docente estará relacionado con la posibilidad de coordinar y gestionar tareas, pero también de reconocer las potencialidades de los demás, de alentar sus iniciativas, de abrir espacios para establecer diálogos y superar las tensiones que la interacción de diferentes racionalidades provoca" (Barrios et al, 2019, p. 87).

Este aspecto alimenta la llama del compromiso y favorece, sin dudarlo, la consolidación de CoP en el sector educativo. Particularmente en el área de matemáticas fue posible revisar variadas investigaciones que develan que en la medida en que una práctica se construya a partir de la negociación entre estudiantes y docentes se constituye un dominio de conocimiento que respalda tales prácticas (Braz y Kato, 2015). Así, la presencia social del maestro al interior de la una CoP de matemáticas es ser aprendiz y enseñante al mismo tiempo (Back y Pratt, 2009). Esto se puede fomentar según Broomes (1989) con actividades "ricas" para el aula, las cuales "permiten establecer conexiones entre distintas áreas del currículum dentro o fuera de las matemáticas, con lo cual amplía su imagen y el desarrollo de significados" (p. 122). Con ello el docente se convierte en alguien que participa del proceso sin ser el protagonista principal, alentando a que el estudiante encuentre la manera de apropiarse de los conocimientos, que al ponerlos en práctica cobraran sentido y relevancia en su aprendizaje.

Por otro lado, Siemos (2009) considera que el aspecto más valioso en la comprensión de las CoP, es su valor para reformular problemas que se convierten finalmente en parte de su repertorio compartido. Por lo tanto, involucrar a los docentes en la exploración del contenido matemático apropiado para su nivel y brindarles a los docentes oportunidades para compartir ideas es un aspecto crítico para el éxito de las CoP (Hodges y Cady, 2013). Desde esta perspectiva las CoP pueden funcionar como laboratorios para la generación de propuestas de investigación en el aula diseñadas en conjunto por profesores e investigadores en las que ambos colectivos aporten sus conocimientos y participen juntos en su desarrollo en las aulas, como lo afirman (Greca y El-Hani, 2015).

\section{En la educación básica primaria}

El número de investigaciones que relacionan las CoP con la educación primaria es notablemente menor comparado con la educación universitaria o con la educación secundaria y media, lo que inmediatamente señala una oportunidad de incrementar la investigación educativa en este nivel académico. Aun así, algunos investigadores han encontrado fortalezas y potencialidades para el mejoramiento de la educación primaria usando CoP.

En la investigación de Encinas (2011) se registra la creación de tres CoP: urbana, rural y comunidades indígenas multigrado, evidenciando que estos colectivos de profesores se encaminaban a visualizar elementos pedagógicos útiles de su práctica docente. Mientras que Aguirre (2015) muestra avances al preconcebir un proceso de investigación donde usó la estrategia de conformación de una CoP con un grupo de docentes interesados en mejorar sus prácticas de aula, resultando en una movilización de los docentes por indagar acerca de los problemas que se les presentan y a "formular de manera conjunta proyectos de mejoramiento beneficiándose de la experiencia compartida y a la vez reconociéndose como capaces de aportar de manera válida soluciones a las problemáticas emergentes" (p. 4).

Por su parte López et al (2011) exponen que los docentes "han respondido mejor a la llamada para unirse y trabajar juntos cuando el referente era la identidad del plantel educativo que cuando lo era la teoría pedagógica" (p. 126). Podría decirse que los docentes de primaria en general sienten una mayor atracción 
hacia el trabajo en equipo cuando este se genera desde su sentido de pertenencia por la institución educativa, que cuando son invitados desde aproximaciones teóricas, es decir, que muestran un mayor aprecio por las apuestas fácticas y contextualizadas por ser más próximas a su ejercicio profesional.

Como lo reafirma Orjuela (2016) al informar que en las CoP los profesores encuentran importante mejorar su práctica contando con sus colegas y comunicando las propias experiencias con sus pares en busca de mayor sentido de pertenencia. Así mismo, "favorecen el desarrollo profesional de los docentes al propiciar múltiples dinámicas de participación y de trabajo colaborativo entre los docentes que permitan mejorar el proceso educativo desde la perspectiva del contexto" (p. 1785). Pérez et al (2013) encuentran que uno de los aspectos de la escuela que más favorece la creación de una CoP de profesores es el liderazgo del director, "lo cual se ve reflejado en la manera como la institución educativa estimula la formación de tales comunidades” ( $p$. 81), es decir que, sin importar la ubicación geográfica de los integrantes es importante contar con un factor cohesivo, como el de un directivo docente. Esta investigación junto con la de Murillo y Pesqueira (2014) se complementan, pues estos afirman que "a través del aprendizaje suscitado en los docentes en las CoP se logra optimizar los recursos y obtener herramientas para detectar y solucionar las áreas de oportunidad prioritarias de la institución" (p. 28). Es claro que las investigaciones realizadas avalan potentemente, cualquier inclinación a utilizar las comunidades de práctica en el sistema educativo.

Estos aspectos se consideran indispensables para desarrollar un sentido de responsabilidad que empuje a los docentes al mejoramiento de las prácticas de aula, pues como indican Giraldo y Quintero (2014) el desempeño académico en pruebas estandarizadas está ligado a la "formación académica y profesional de los docentes, a la innovación y a la investigación constante que genera diferentes visiones y amplia el bagaje conceptual al docente" (p. 89), y por ende mejores oportunidades de aprendizaje a los estudiantes. Así, el enriquecimiento está al alcance de los profesores en general y en particular de los profesores de primaria, que, dadas las circunstancias, en la mayoría de los casos manifiestan su deseo por incrementar el aprendizaje de sus clases, en particular, las de matemáticas.

\section{Conclusiones}

La presente revisión bibliográfica permitió visualizar las fortalezas y las oportunidades existentes que emergen al usar las CoP en el sistema educativo, así como los avances en su implementación y puesta en marcha en los diferentes niveles y contextos analizados.

En consecuencia, se puede afirmar que este tipo de estructura organizacional está siendo utilizada como plataforma para impulsar el mejoramiento de los procesos educativos al interior de las universidades. Esto, claramente se hace explícito en la promoción de un ambiente de confianza que estimule la colaboración entre pares; en la generación de espacios reflexivos como base para la cualificación de las prácticas pedagógicas; en la correspondiente gestión del conocimiento incentivado por el intercambio interdisciplinar de saberes; en la configuración de repertorios compartidos de prácticas revisadas y reflexionadas conjuntamente, en la promoción de metodologías investigativas de carácter cualitativo que reconocen el sujeto como agente activo del problema y en su énfasis de servir como una estructura por la cual se articule el proceso mismo de ejecución de las intenciones de mejoramiento y transformación incubadas en su interior.

Por su parte, en la educación secundaria y media el enfoque que se presenta en el uso de CoP está ligado al mejoramiento de la enseñanza, al fortalecimiento disciplinar y al compartir estrategias metodológicas que se puedan replicar en las prácticas de aula particulares. Así mismo, se impulsan las dinámicas reflexivas sobre el desempeño docente, la posibilidad de compartir saberes al alcance de todos los miembros de las CoP generando un compromiso mutuo y un sentido de identidad que emerge y apalanca la consecución de metas comunes. Además, se fortalecen los canales de comunicación que proporcionan el nivel de entendimiento necesario para establecer una negociación de significados como base para la comprensión del contexto educativo. Por lo tanto, es primordial contar con liderazgos activos al interior y alrededor de la CoP con el fin de apoyar los procesos de estructuración requeridos para optimizar el desarrollo profesional de los docentes.

En la educación básica primaria las investigaciones sobre CoP son mucho menores que en los dos anteriores niveles. Una conjetura para que ello suceda es tal vez, la falta de un vínculo entre los diferentes niveles educativos que le permita al docente de primaria imbuirse en una dinámica investigativa. Otro aspecto es que la enseñanza en la educación básica primaria es una situación de tanta dedicación que al profesor de este nivel no le queda el tiempo necesario para suscribirse a grupos de investigación. Sin embargo, en los artículos revisados es posible notar una tendencia marcada por privilegiar los elementos prácticos de los que puedan 
beneficiarse los miembros de una CoP. Es decir, que los docentes de básica primaria privilegian los espacios de formación si estos son mediados a través de actividades prácticas que puedan replicarse en el aula de clases.

Sin embargo, es también importante mencionar que en los tres niveles educativos analizados se evidencian una serie de características compartidas, a saber:

- La reflexión como mecanismo de autorrevisión.

- El intercambio de saberes como un proceso simbiótico de colaboración y cooperación en equipo.

- El desarrollo de la confianza en el otro y en la CoP, como un reconocimiento de un compromiso mutuo y una empresa conjunta.

- El énfasis en la utilización de una metodología cualitativa, claramente diseñada para procesos de transformación que promueve el mejoramiento de las de las circunstancias iniciales que le dieron vida.

- La estimulación del liderazgo entre los miembros de la CoP para colimar esfuerzos y voluntades alrededor de las intenciones del grupo.

Para concluir, en palabras de Murcia (2014) "lo que define el éxito o fracaso de las instituciones educativas exitosas en comparación con aquellas que no lo son, es la configuración de comunidades de práctica en su interior, las cuales generan unas formas comunes de asumir el ser humano, la sociedad y el mundo" ( $p$. 45). Es decir que, la estructuración de una CoP de docentes estimularía el mejoramiento propio y contextual de las condiciones que promueven la calidad de la enseñanza en un establecimiento educativo. Pues, de acuerdo Hernández y Flores (2013) en el caso de la educación matemática, "una CoP la constituye un grupo de profesores que aprenden en su actividad, negocian significados sobre la acción docente, generan oportunidades de desarrollo profesional y mejoran la enseñanza en contextos particulares" (p. 106). Así, se visualiza una oportunidad de aportar al conocimiento desde una investigación cualitativa que busque determinar los efectos de cultivar una CoP al interior de una institución educativa con docentes de la educación básica primaria cuyo propósito sea el mejoramiento de la enseñanza de las matemáticas mediante la reflexión entre pares y la generación de lazos de confianza que impulsen su desarrollo.

Sin duda, dichas características prometen excelentes resultados para la implementación de una CoP en una organización educativa. Sin embargo, sus efectos podrían ser mayores, como dice Vasco (2015) si se lograse una verdadera articulación entre el nivel de preescolar y el nivel de primaria; entre el nivel de primaria y el nivel de secundaria; entre el nivel de secundaria y media y la universidad, se formarían puentes de entendimiento curricular y profesional que posean una visión común y que produzcan unos resultados acordes a las necesidades sociales del contexto. Por otro lado, si se contase con que los directivos docentes llevaran a cabo su rol de líderes pedagógicos, podrían fortalecerse las iniciativas profesorales que tengan como principio fundamental el trabajo colaborativo en una empresa conjunta cuyo compromiso mutuo sea tal que el repertorio compartido emerja para el beneficio de todos. Es necesario entonces, avizorar nuevas líneas investigativas que complementen lo investigado y se expanda hacia otras áreas del conocimiento, así como a otros niveles educativos (p. ej.: el preescolar, la educación de adultos), y a procesos como el aprendizaje, la programación curricular, la organización escolar, los ambientes de clase, la gestión comunitaria y tantos, como el contexto lo permita y requiera.

\section{Referencias Bibliográficas}

Agudelo, M. y Lovera, C. (2017). Arqueología y genealogía de las comunidades de práctica en Colombia. Revista Aletheia, 11(2), p. 37-62.

Aguirre, C. (2015). Desarrollo De Competencias En La Educación Primaria: La Conformación De Una Comunidad De Práctica. Ponencia: Congreso nacional de investigación educativa.

Almánzar, V. (2013). Los estudios Generales en comunidades de Práctica. Instituto tecnológico de Santo Domingo, INTEC. República Dominicana.

Anguita, R; Rubia, B.; Jorrín, I.; Ruíz, I.; Villagrá, S. (2006). Creando comunidades de práctica educativa en la universidad. Ponencia. Un estudio de casos en la asignatura de nuevas tecnologías aplicadas a la educación. Universidad de Valladolid. Facultad de Educación y Trabajo Social, Campus Miguel. 
Ávalos, B. (2011). El liderazgo docente en comunidades de práctica. Revista Educar, 47 (2) 237 - 252.

Aydee, R. (2015). Subjetividades docentes en la universidad pública colombiana. Comunidades de práctica a propósito de sus narraciones. Revista Colombiana de Educación, 68, 229-263.

Back, J. y Pratt, N. (2009). Spaces to discuss mathematics: Communities of practice on an online discussion board. For the learning of mathematics Review, 27(2) 34 - 39.

Barragán, D. (2015). Las Comunidades de Práctica (CP): hacia una reconfiguración hermenéutica. Revista Franciscanum 163, 155-176.

Barrios, D; Zuluaga, Z; García, M; Gómez, F; Santamaría, A; Castro, L y Sánchez, A. (2019). Comunidades de práctica como marco comprensivo del talento docente. Magis. Revista Internacional de Investigación en Educación, 11 (23), p. 75-94. doi: 10.11144/Javeriana.m11-23.cpmc.

Bozu, Z y Imbernon, F. (2009). Creando comunidades de práctica y conocimiento en la Universidad: una experiencia de trabajo entre las universidades de lengua catalana. Revista de Universidad y Sociedad del Conocimiento (RUSC). 6 (1) UOC. Consultado el 16 de abril de 2019 en www.raco.cat/ index.php/RUSC/article/download/129746/179181.

Braz, B. y Kato, L. (2015). Constituição de Comunidades de Práticas Locais e o Ambiente de Aprendizagem da Modelagem Matemática: algumas relações. Bolema: Boletim de Educação Matemática, 29(52), p. 613-636. https://doi.org/10.1590/1980-4415v29n52a10.

Broomes, D. (1989). Using goals to construct useful forms of school mathematics. Paris.

Bustos, M.; Moreno, J. (2008). Sistematización de prácticas. Una reflexión al interior de la comunidad de práctica del eje de problemas y pensamiento matemático avanzado. Noveno encuentro Colombiano de Matemática Educativa. Universidad Popular del Cesar -UPC-, Valledupar, Colombia.

Camargo, L. (2010). Descripción Y Análisis De Un Caso De Enseñanza Y Aprendizaje De La Demostración En Una Comunidad De Práctica De Futuros Profesores De Matemáticas De Educación Secundaria. Tesis Doctoral. Universidad de Valencia. Valencia (España).

Castellanos, M. (2015). Las comunidades de práctica, como una estrategia para mejorar la práctica docente. Revista de Ciencias de la Educación Academicus. 1(7). Oaxaca, México.

Chacón, M. (2006). La reflexión y la crítica en la formación docente. Revista Educere, 10 (33) 335-342

Cheek, J; Corlis, M y Radoslovich, H. (2009). Connecting what we do with what we know: building a community of research and practice. International Journal of Older People Nursing 4, 233-238. Doi: 10.1111/j.1748-3743.2009.00181.x

Conde, L; Parada, S y Fiallo, J. (2017). Reflexiones en comunidad de práctica sobre Triángulos imposibles en clase de matemáticas. Educação e Pesquisa, 43, (2) 453-466. Doi: http://dx.doi.org/10.1590/ $\underline{\text { S1517-9702201611150509. }}$.

Cordero, S. (2013). ¿Cómo se forman los docentes Universitarios? Un estudio Desde la perspectiva de las Comunidades de práctica en Clases innovadoras de física. IX congreso internacional sobre investigación en didáctica de las ciencias. Girona, 9-12 de septiembre de 2013.

Córdoba, M. (2013). Comunidades de Práctica como estrategia de formación docente para el fortalecimiento de los estudios generales: el caso del INTEC. V Simposio Internacional de Estudios Generales. 6, 7 y 8 de noviembre de 2013, en la Universidad de Puerto Rico Recinto de Río Piedras. Red Internacional de Estudios Generales (RIDEG). Recuperado el 24 de febrero de 2019 en http://www.rideg.org/wp-content/uploads/2014/04/Comunidades-de-Pr\%C3\%A1ctica-como-estrategia-de-formaci\%C3\%B3n-docente-para-el-fortalecimiento-de-los-estudios-generales-el-caso-del-INTEC.pdf 
Cyrino, M. (2016). Mathematics Teachers' Professional Identity Development in Communities of Practice: Reifications of Proportional Reasoning Teaching. Bolema: Boletim de Educação Matemática, 30(54), 165-187. https://doi.org/10.1590/1980-4415v30n54a08.

Da Silva, H. (2010). Uma Caracterização do Centro de Educação Matemática - CEM (1984-1997) como uma Comunidade de Prática de Formação Continuada de Professores de Matemática. Boletim de Educação Matemática, 23 (35) 185-218

Edwars, M y Rebollo, C. (2010). Propuestas de formación docente basada en la construcción de comunidades de práctica: experiencias en diferentes contextos educativos de España y Uruguay. Congreso Iberoamericano de Educación Metas 2021. Buenos Aires, Argentina. 13, 14 y 15 de septiembre de 2010. Recuperado de https://www.oei.es/Educacion/metas2021/documento-final .

Ekici, D. (2017). The effects of online communities of practice on pre-service teachers' critical thinking dispositions. Eurasia Journal of Mathematics Science and Technology Education, 13(7):38013827. DOI 10.12973/eurasia.2017.00759a

Encinas, A. (2011). El Colectivo Docente Una Comunidad De Práctica Situada y Negociada. Departamento de Investigaciones Educativas, CINVESTAV-IPN/ Instituto de Formación Docente de Estado de Sonora. XI Congreso Nacional de Investigación Educativa / 15. Procesos de Formación / Ponencia.

García, J., Greca, I y Meneses, J. (2008). Comunidades virtuales de práctica para el desarrollo profesional docente en Enseñanza de las Ciencias. Revista Electrónica de Enseñanza de las Ciencias, 7 (2) 439-462.

Giraldo, A., Quintero, L. (2014). Pruebas Saber: Una Perspectiva Desde La Educación Matemática De Los Docentes De Primaria. Tesis de maestría en Educación Matemática. Universidad de Medellín. Antioquia.

Giraldo, L y Atehortúa, L. (2010). Comunidades de práctica, una estrategia para la democratización del conocimiento en las organizaciones, Una reflexión. Revista Ingenierías Universidad de Medellín, 9 (16) 141-150.

Godói de Sousa, E., Eiko, L. (2013). Comunidades de Práctica: Una Innovación en la Gestión del Conocimiento. Journal of Technology Management \& Innovation. 8, 11-11

González, D. (2014). Comunidad de práctica: un espacio para la constitución de la identidad del profesor de estadística. En Andrade, Luisa (Ed.), Memorias del I Encuentro Colombiano de Educación Estocástica, p. 191-197. Bogotá: Asociación Colombiana de Educación Estocástica.

González, R.; Castañeda, F.; Torres, M.; Banda, R.; Vargas, R. y Ruiz, F. (2013). Colaboración en comunidad de práctica para el Desarrollo profesional del profesor. Revista de Medios y Educación 42,103113.

Graven, M. (2004). Investigating mathematics teacher learning within an in-service community of practice: the centrality of confidence. Educational Studies in Mathematics, 57, 177-211.

Greca, I. y El-Hani, C. (2015). Docentes e investigadores en comunidades virtuales de práctica para el desarrollo profesional docente y la mejoría de la enseñanza de las ciencias. Revista de Enseñanza de la Física. 27(1) 7-18.

Hernández, A. y Flores, R. (2013). Caracterización de una comunidad de práctica orientada al uso de la matemática en la enseñanza de la ingeniería. Innovación educative, 13(62), 101-119.

Hodges, T. y Cady, J. (2013). Blended-format professional development and the emergence of communities of practice. Mathematics Education Research Journal, 25(2). 299-316.

Jho, H., Hong, O., y Song, J. (2016). An Analysis of STEM/STEAM Teacher Education in Korea with a Case Study of Two Schools from a Community of Practice Perspective. Eurasia Journal of Mathematics, Science and Technology Education, 12(7), p. 1843-1862. https://doi.org/10.12973/eurasia.2016.1538a 
Jubert, A. (1999). Developing an Infraestructure for Communities of Practice. In Proceedings of the 19th International Online Meeting, ed. B. Mckenna. Oxford: Learned Information.

Cubero, M. J. L., \& Sazatornil, J. L. L. (2013). Aprendizaje colaborativo en comunidades de práctica en entornos de exclusión social. Un análisis de las interacciones1 Collaborative learning in communities of practice in contexts of social exclusion. An analysis of the interactions. Revista de educación, 362, 402-428.

López, J., Sánchez, M. y Altopiedi, M. (2011). Comunidades profesionales de práctica que logran sostener procesos de mejora institucional en las escuelas. Revista de Educación, 356. 109-131.

Mayorga, M. (2014). Las comunidades de práctica y el trabajo colaborativo desde los AVA: Alternativa para la construcción del pensamiento social. Comunic@Red, 1(1) 257-278.

McDermott, R. (1999). Why Information Technology Inspired But Cannot Deliver Knowledge Management. California Review Management, 4I(4) 103-117.

Morales, A. y Flores, R. (2013). Caracterización de una comunidad de práctica orientada al uso de la matemática en la enseñanza de la ingeniería. Revista Innovación Educativa, 13 (62) 101-119. .

Morueta, R.; Boza, A. y Aguaded, J. (2009). Comunidades De Práctica En La Red: Indicadores Y Condiciones Para Su Desarrollo. RevistaTendencias Pedagógicas, 14, 303-324

Murcia, C. y Bustos, J. (2000). Las comunidades de práctica como herramientas para construir conocimiento estadístico. Décimo encuentro colombiano de Matemática Educativa. Universidad Distrital Francisco José de Caldas.

Murcia, N. (2014). Comunidades de práctica: Huellas en el currículo de la Escuela Activa Urbana en Manizales. Revista Criterios, 21(1), p. 23-48.

Murillo, G. y Pesqueira, N. (2014). Un estudio de caso: La innovación de la estructura organizacional a través de comunidades de práctica en Educación primaria en el programa de escuelas de tiempo completo, en Hermosillo Sonora. III Feria de Posgrado en Educación. Ponencia.

Orjuela, C. (2016). Memorias, Séptimo Congreso Internacional sobre Formación de Profesores de Ciencias. Revista Tecné, Episteme y Didaxis: TED.

Ortega, D y Hernández, P. (2015). Evaluación formativa en comunidades de práctica. Avances de investigación en la mejora de la educación en la formación de docentes, Tomo III. Universidad Autónoma Indígena de México. Sinaloa, México.

Parada, S. (2011). Reflexión y acción en comunidades de práctica: Un modelo de desarrollo profesional. Tesis Doctoral. Centro De Investigación Y De Estudios Avanzados Del IPN. Ciudad de México, Distrito Federal (México).

Pérez, A., Perea-Henze, I. y Flores, M. (2013). Comunidades de práctica de profesores de escuelas primarias públicas: un estudio de caso colombiano. Actualidades Pedagógicas, 62, 69-84.

Pérez, J. (2011). Comunidades de práctica como sistemas emergentes de aprendizaje. Actualidades Pedagógicas, 58, 15-27.

Perrenoud, P. (2011). Desarrollar la Práctica Reflexiva en el oficio de Enseñar. Barcelona: Editorial Grao.

Pyrko I; Dörfler, V y Eden, C. (2016). Thinking together: What makes Communities of Practice work? Human Relations; 70(4), 389-409

Ramos, W. y Manrique, A. (2015). Comunidade de Prática de Professores que Ensinam Matemática como Espaço de Negociações de Significados sobre a Resolução de Problemas. Bolema, Rio Claro (SP), 29 (53) 979-997. DOI: http://dx.doi.org/10.1590/1980-4415v29n53a10 .

Ravanal, E. (2016). Coordinación y aprendizaje en una comunidad de práctica con profesores universitarios REXE. Revista de Estudios y Experiencias en Educación, 15(29) 15-28. 
Rodrigues, W; Manrique, A. (2015). Comunidade de Prática de Professores que Ensinam Matemática como Espaço de Negociações de Significados sobre a Resolução de Problemas. Boletim de Educação Matemática, 29 (53) 979-997

Rodríguez, J. (2007). Comunidades virtuales, práctica y aprendizaje: elementos para una problemática. Revista Electrónica Teoría de la Educación. Educación y Cultura en la Sociedad de la Información, 8 (3). Recuperado de http://www.redalyc.org/articulo.oa?id=201017307002 .

Roig, G. y Rivera, A. (2008). La comunidad de práctica como modalidad de Desarrollo profesional de maestros en Investigación acción: dirigido hacia el Aprendizaje con entendimiento de conceptos Científicos y matemáticos. Memorias de las comunidades de práctica en investigación acción de ALACIMA: años académicos 2006-2007 y 2007-2008, p. 25 - 39. Universidad de Puerto Rico - Recinto de Humacao.

Sabulsky, G. (2016). Comunidades de Práctica para el mejoramiento de la enseñanza de las Ciencias Económicas. Experiencias de la comunidad de prácticas para el mejoramiento de la enseñanza de las ciencias económicas: juegos y actividades interactivas en el aula. Compilado por Funes, M.; Guardiola, M. - 1a ed. - Córdoba: Asociación Cooperadora de la Facultad de Ciencias Económicas de la U.N.C.

Sanz, M. y Pantoja, A. (2015). Formación permanente del profesorado en las comunidades de práctica. Aula de Encuentro, 1(17) 105-130.

Schön, D. (1982). El profesional reflexivo. Cómo piensan los profesionales cuando actúan. BA: Paidós.

Siemos, D. (2009). Developing mathematics knowledge keepers - issues at the intersection of communities of practice. Eurasia Journal of Mathematics, Science \& Technology Education, 5(3), 221-234.

Solomon, Y., Eriksen, E., Smestad, B., Rodal, C. \& Bjerke, A.H. (2015). Prospective teachers navigating intersecting communities of practice: early school placement. Journal of Mathematics Teacher Education, 20(2), 141-158. doi:10.1007/s10857-015-9327-6.

Torres, R. y Soriano, A. (2015). Creación de comunidades de práctica y conocimiento, como metodología didáctica: caso SocialOVTT en la disciplina de Relaciones Públicas. Revista Internacional de Tecnología, Conocimiento y Sociedad, 4(2), p. 169-179.

Trust, T. (2015). Deconstructing an Online Community of Practice: Teachers' Actions in the Edmodo Math Subject Community. Journal of Digital Learning in Teacher Education, 31(2), 73-81. Disponible en: http://www.tandfonline.com/doi/abs/10.1080/21532974. 2015.1011293.

Valdés, M. (2010). Las Comunidades de Práctica de Profesores como un Medio para la Mejora de su Práctica Docente. El Caso del Grupo Escuelas de Clase Mundial en el Estado de Nuevo León, México. Tesis de Doctorado. Tecnológico de Monterrey, Escuela de Graduados en Educación. Monterrey, México.

Vallín, M. (2013). Hacia la autoformación permanente de los docentes en las comunidades de práctica. Obra Digital, 4, 56-74.

Vargas, J.; Martinez, M. y González, K. (2019). Comunidad De Práctica En Inacap: Un Espacio Para EI Mejoramiento De Prácticas Pedagógicas. Acta latinoamericana de matemática educativa..

Vargas, J.; Orozco, A,; Escudero, R.; Crespo, J.; Castro, D.; Lobo, R.; Cervantes, G. y Jimenéz, G. (2016). Sábado del docente: comunidad de práctica para la investigación e innovación en la enseñanza de las ciencias. Tecné, Episteme y Didaxis: TED.

Vasco, C. (2015). Diez retos para la educación colombiana para el 2025. La Calidad de la Educación bajo la Lupa. Bogotá: Editorial magisterio

Vásquez, S. (2011). Comunidades de práctica. EDUCAR. 47(1) 51-68

Wenger, E. (2001). Comunidades de práctica: aprendizaje, significado e identidad. Paidós. 
Wenger, E., McDermott, R., Snyder, W. (2002). Cultivating Communities of Practice: A Guide to Managing Knowledge. Massachusetts: Harvard Business School Press.

Zea, L., y Acuña, L. (2017). Comunidades de saber y práctica pedagógica. Aula Urbana, 105, 3-5. Recuperado a partir de https://revistas.idep.edu.co/index.php/mau/article/view/1703 